\title{
Spectral modeling of the three spectral states of the Galactic microquasar GRS 1915+105
}

\author{
M. A. Sobolewska and P. T. Życki \\ Nicolaus Copernicus Astronomical Center, Bartycka 18, 00-716 Warsaw, Poland \\ e-mail: malsob@camk.edu.pl \\ Received 3 June 2002 / Accepted 7 January 2003

\begin{abstract}
We have analyzed Rossi X-ray Timing Explorer spectral data of the Galactic micro-quasar GRS $1915+105$ in its various spectral states, as defined by Belloni et al. (2000). In states A and B the spectra are dominated by a strong soft thermal component, accompanied by a weak harder tail. The soft component is rather complex and cannot be described as a simple accretion disk emission. Relativistic effects in Kerr metric contribute to the complexity of the soft component but are not sufficient to fully account for it. As found previously, state $\mathrm{C}$ spectra are dominated by a Comptonized component, with small contribution from disk photons. The X-ray reprocessed component is highly significant in those spectra and, in contrast to the usual hard state spectra from accreting black holes, it is highly ionized.
\end{abstract}

Key words. accretion, accretion disks - stars: individual: GRS 1915+105 - X-rays: binaries

\section{Introduction}

Ever since its discovery by Castro-Tirado et al. (1994), the transient Galactic source GRS $1915+105$ has been a subject of intense observational and theoretical work. The source shows a number of remarkable features, most spectacular of which are ejections of relativistic jets with apparent super-luminal motion (Mirabel \& Rodriguez 1994). It displays a large variety of behaviour in the X-ray band (dramatic flux and spectral variability on time scales from miliseconds to months), most likely reflecting violent phenomena occurring in the inner accretion flow.

Large amount of data collected by various X-ray observatories indicate that the source stays permanently in a soft spectral state (see, e.g., Nowak 1995 for a characteristics of the various spectral/timing states). That is, its spectral energy distribution is dominated by the $1-10 \mathrm{keV}$ band, even though the ratio of soft thermal (temperature $\sim 1 \mathrm{keV}$ ) emission to harder Comptonized continuum does vary substantially. The source never seems to have been in the hard spectral state, which is more typical for black hole binaries with energy distribution peaking at $\sim 100 \mathrm{keV}$ (see Poutanen 1998 for review).

A characteristic feature of GRS $1915+105$ is its strong variability, of both flux (count rate) and energy spectra. Among the variety of light curves and color-color diagrams certain patterns can be found. These were studied in detail by Belloni et al. (2000; hereafter B00), using RXTE data, who attempted to classify the behaviour of the source. They divided the lightcurves

Send offprint requests to: P. T. Życki,

e-mail: ptz@camk.edu.pl into 12 classes based on the color-color diagrams and count rates, and found that the lightcurves from each class can be decomposed into only 3 basic spectral states (A, B, C in the nomenclature of $\mathrm{B} 00$ ). The strongly variable lightcurves of the source could be explained in terms of rapid switches between these three states.

There have been previous attempts to model the X-ray spectra of GRS $1915+105$ in the B and C spectral states. Vilhu et al. (2001) analyzed RXTE data (PCA/HEXTE instruments, in the range of $2-60 \mathrm{keV}$ ) and concluded that spectra of GRS $1915+105$ can be described with the model consisting of a disk blackbody and a Comptonized component with Compton reflection. Zdziarski et al. (2001) presented an analysis of simultaneous observations of GRS 1915+105 in states B and $\mathrm{C}$ by RXTE and CGRO/OSSE instruments. They found that the broad-band spectra required non-thermal Comptonization of soft photons, since the data do not show any break up to at least $600 \mathrm{keV}$. Low temperature, thermal Comptonization was also needed to correctly model (together with blackbody component) the soft X-ray part $(E<10 \mathrm{keV})$, thus the best final model invoked a hybrid plasma (Coppi 1999). Vadavale et al. (2001) focused on a study of the source during the steady low-hard state (class $\chi$, state $\mathrm{C}$ ), which they further subdivided into radio-loud $\left(\chi_{\mathrm{RL}}\right)$ and radio-quiet $\left(\chi_{\mathrm{RQ}}\right)$ ones. From their analysis followed that the spectrum of GRS $1915+105$ in state $\mathrm{C}$ can be described as a superposition of disk blackbody and Comptonized component, and an additional power low component in the case of the $\chi_{\mathrm{RL}}$ state. This additional power low was interpreted as an emission from the base of a jet. Analysis of spectral features due to iron emission/absorption in 
the 5-9 keV band was performed by Martocchia et al. (2002) using BeppoSAX data of the source in state $\mathrm{C}$, according to their interpretation. They found that the $\mathrm{Fe} \mathrm{K}_{\alpha}$ line is broad and it has the characteristic asymmetric profile indicating its origin from a rotating accretion disk.

In all above mentioned analyses, disk blackbody or blackbody component is used to (fully or partially) describe the soft emission from the disk. The diskbb model reproduces the shape of the disk spectrum quite accurately unless the relativistic corrections are extreme. However, the numerical values of the parameters do not easily translate into physical parameters (Merloni et al. 2000). These can be estimated from a relativistic model, which includes radiative transfer effects, at least approximating them by introducing the color temperature correction (Shimura \& Takahara 1995).

Therefore, in this paper we analyse data from all three spectral states of GRS 1915+105, applying the same set of models to all the data. The models include one for emission from an accretion disk around a maximally rotating Kerr black hole.

\section{Data selection and reduction}

The log of PCA/HEXTE observations used for our analysis is given in Table 1. The data were extracted from public archive at HEASARC/GSFC and reduced with the FTOOLS software package (version 5.0).

Based on B00 we attempted to extract spectra corresponding to "pure" spectral states A, B and C. We have two observations from class $\chi$ (pure state $\mathrm{C}$ ), one observation from class $\lambda$ from which we extract a period corresponding to state B based on the $\mathrm{X}$-ray color-color diagrams and lightcurves appearance (see B00 for details), one observation from class $\theta$ which provides state $\mathrm{A}$ spectra, and two observations from class $\beta$ - further examples of state A, B, and C spectra.

For the spectral analysis, PCA Standard 2 mode data (PCU 0-2, top layer only) and HEXTE Archive Mode (both clusters) configuration are used. The energy range is $3-20 \mathrm{keV}$ and 20 $100 \mathrm{keV}$, correspondingly. We assume systematic errors in the PCA data at the level of $1 \%$ due to the uncertainty of response matrices. Standard background subtraction and dead-time correction procedures are applied.

The value of the hydrogen column density, $N_{\mathrm{H}}$, is allowed to vary during the fit. We assume that the source inclination is $i=70^{\circ}$, distance $d=12.5 \mathrm{kpc}$ (Mirabel \& Rodriguez 1994) and central black hole mass $M=14 M_{\odot}$ (Greiner et al. 2001). We allow for free relative normalization of HEXTE data with respect to PCA data. Analysis was performed in XSPEC ver. 11 (Arnaud 1996).

\section{Models}

The disk soft thermal emission will be described by the simple disk blackbody model (Mitsuda et al. 1984; implemented as diskbb) in XSPEC, or a relativistic accretion disk model for Kerr geometry (Ebisawa et al. 2001). The KerrD model is computed for the maximally rotating black hole ( $a=0.998$; Thorne 1974). It assumes blackbody spectra at each radius with
Table 1. The log of observations. The letters $I, K$ stand for observations ID 10408-01, 20402-01, respectively (B00). The third column indicates which state spectra were extracted from the data.

\begin{tabular}{ccc}
\hline \hline Observation ID & Class & State \\
\hline$K-44-00$ & $\beta$ & $\mathrm{A}, \mathrm{B}, \mathrm{C}$ \\
$K-45-03$ & $\beta$ & $\mathrm{A}, \mathrm{B}, \mathrm{C}$ \\
$K-45-02$ & $\theta$ & $\mathrm{A}, \mathrm{B}$ \\
$I-38-00$ & $\lambda$ & $\mathrm{B}$ \\
$K-05-00$ & $\chi^{2}$ & $\mathrm{C}$ \\
$I-42-00$ & $\chi^{4}$ & $\mathrm{C}$ \\
\hline
\end{tabular}

the color temperature correction constant with radius and convolves them with the transfer function describing photon propagation, as computed by Laor (1991). We fix the black hole mass, source distance and accretion disk inclination at the values indicated above, and allow only the inner radius of the disk (in units of $R_{\mathrm{g}} \equiv G M / c^{2}$ ) and the mass accretion rate, $\dot{M}$ to be free parameters. In particular, the model does not have an additional free normalization.

The Comptonized component will be described by the thComp model, based on a solution of the Kompaneets equation (Zdziarski et al. 1996), or by the compPS model of Poutanen \& Svensson (1996). The latter does not assume the diffusion approximation but solves for each scattering order independently. The compPS model can be used with purely thermal, purely non-thermal or a hybrid electron energy distribution (Coppi 1999). The seed photons will be assumed to have a disk blackbody spectrum and the comptonizing cloud is assumed to be a sphere. The code also consistently computes the un-scattered fraction of seed photons.

For the X-ray reprocessed component we will use the relrepr model (Życki et al. 2001), combining the Comptonreflected continuum (Magdziarz \& Zdziarski 1995; Done et al. 1992) with the $\mathrm{Fe} \mathrm{K}_{\alpha}$ line computations of Życki \& Czerny (1994). The model computes self-consistently the strength of the line for a given primary continuum spectrum, amplitude of the Compton-reflected component, $R$, ionization parameter, $\xi=4 \pi F_{\mathrm{X}} / n$, and $\mathrm{Fe}$ abundance, and it also allows for relativistic effects to be applied to both components (using the prescription from Fabian et al. 1989). The amplitude $R$ is defined as $\Omega /(2 \pi)$, where $\Omega$ is the solid angle subtended by the reprocessor from the $\mathrm{X}$-ray source.

\section{Results}

\subsection{State B spectra}

We begin our analysis with the brightest state, B. First, we try modeling the soft part of the spectrum with disk blackbody, and the hard one with non-thermal Comptonization (we apply compPS model to the data). The latter is motivated by the reports, where the source spectrum in state $\mathrm{B}$ and $\mathrm{C}$ was found to extend to $\geq 600 \mathrm{keV}$ without a break or cutoff (Zdziarski et al. 2001). We assume that the seed photons for Comptonization have the same disk blackbody shape and temperature. The reprocessed component is also included in the model. This model gives unacceptable fits, $\chi_{v}^{2} \sim 2$, with residuals up to 10 per cent 
Table 2. Values of $\frac{\chi^{2}}{\text { d.o.f. }}\left(\chi_{v}^{2}\right)$ for various models fitted to the data.

\begin{tabular}{llllll}
\hline \hline State & Obs. Id & $(1)$ & $(2)$ & $(3)$ & $(4)$ \\
\hline \multirow{3}{*}{$\mathrm{C}$} & $K-44-00$ & $\frac{114.5}{101}(1.14)$ & - & - & - \\
& $K-45-03$ & $\frac{84.2}{101}(0.83)$ & - & - & - \\
& $K-05-00$ & $\frac{83.8}{96}(0.87)$ & - & - & - \\
& $I-42-00$ & $\frac{102.9}{99}(1.04)$ & - & - & - \\
\hline \multirow{4}{*}{$\mathrm{A}$} & $K-44-00$ & 4.8 & $\frac{45.6}{65}(0.70)$ & $\frac{44.6}{66}(0.68)$ & $\frac{43.8}{66}(0.66)$ \\
& $K-45-03$ & 3.4 & $\frac{75.1}{61}(1.23)$ & $\frac{73.7}{62}(1.19)$ & $\frac{79.9}{62}(1.29)$ \\
& $K-45-02$ & 3.4 & $\frac{54.1}{65}(0.83)$ & $\frac{54.8}{66}(0.83)$ & $\frac{63.3}{66}(0.96)$ \\
\hline \multirow{3}{*}{ B } & $K-44-00$ & 2.7 & $\frac{51.8}{66}(0.79)$ & $\frac{47.1}{67}(0.70)$ & $\frac{43.3}{67}(0.65)$ \\
& $K-45-03$ & 1.6 & $\frac{85.4}{85}(1.00)$ & $\frac{83.5}{86}(0.97)$ & $\frac{80.0}{86}(0.93)$ \\
& $I-38-00$ & 2.3 & $\frac{88.2}{95}(0.93)$ & $\frac{85.4}{96}(0.89)$ & $\frac{81.0}{96}(0.84)$ \\
\hline
\end{tabular}

Models (2), (3) and (4) fitted to state A spectra contain an additional Gaussian line.

Fitted models:

(1) compPS - comptonized disk blackbody.

(2) thComp + compPS - two comptonized disk blackbody spectra.

(3) bbody +compPS - comptonized disk blackbody with an additional blackbody.

(4) KerrD + compPS - Kerr metric disk spectrum and a comptonized disk blackbody.

In all fits the compPS model includes the reprocessed component.

(Model 1 in Table 2). A good description of the data is provided by models including a more complex shape of the soft component. Motivated by earlier results (see Życki et al. 2001; Wilson \& Done 2001 and references therein) we test two phenomenological descriptions of the soft component: (i) with an additional Comptonized disk blackbody and (ii) an additional blackbody. (Note that a certain fraction of the seed disk blackbody photons always contributes to the soft component due to modeling the hard part of the spectrum with compPS). Both these complex models give significant improvement compared to Model 1, with best fits of comparable quality, as shown in Table 2 (Models 2 and 3).

A possible physical reason for the complexity may be the relativistic effects expected from an inclined accretion disk. Therefore we also try (iii) the KerrD model for the soft component (with non-thermal compPS for the hard tail, as before). The quality of the fits is similar for all three models (Model 4 in Table 2). The relativistic effects are quite pronounced at the high inclination of the source, $i \approx 70^{\circ}$ (see Ebisawa et al. 2001; Gierliński et al. 2001), and the Doppler-boosted disk emission fits well the observed high energy cutoff of the soft component. In the best fit for $K-44-00$ the inner disk radius $R_{\text {in }}=3.80_{-2.56}^{+2.20} R_{\mathrm{g}}$, for $i=70^{\circ}$ and $d=12.5 \mathrm{kpc}$. With the more recently determined $i=66^{\circ}$ and $d=11 \mathrm{kpc}$ (Fender et al. 1999) the inner radius is $R_{\text {in }}=2.25_{-1.01}^{+1.0}$. In both cases $R_{\text {in }}$ is consistent with the last stable orbit at $1.24 R_{\mathrm{g}}$, and a large inner hole in the disk is ruled out. A good fit $\left(\chi^{2} / v=46.3 / 72\right)$ can be obtained with the disk emission model in Schwarzschild metric, however a small mass of the black hole, $M \approx 4 M_{\odot}$ is required in this case, in order to account for the high observed disk temperature. Thus, if the soft component is modeled as a relativistic disk emission, a Kerr (rotating) black hole is implied by our result.
We note once again that some seed disk blackbody photons do contribute to the soft component (Fig. 1) since the compPS model computes self-consistently the un-scattered fraction of the seed photon flux. Without this component the fit is worse by $\Delta \chi^{2}=21$ (for $K-44-00$ ), therefore we stress that the relativistic effects may not be the only factor contributing to the complexity of the soft component. However, given the high inclination of the accretion disc, their a priori importance is obvious. The mass accretion rate is $7.5 \times 10^{18} \mathrm{~g} \mathrm{~s}^{-1}$ for $K-44-00$ data (Table 3; and up to $2.6 \times 10^{19} \mathrm{~g} \mathrm{~s}^{-1}$ for other data). We note that both $\dot{M}$ and $R_{\text {in }}$ would be different for different value of the black hole angular momentum (see discussion in Gierliński et al. 2001; recall that $a=0.998$ is assumed in our paper), thus our result can only be regarded as a qualitative demonstration that relativistic effects can partially account for apparently complex soft component in this spectral state.

The seed photon temperature for the non-thermal Comptonization was a free parameter in these fits, but the best fit values are similar to the obtained maximum temperatures of the Kerr disk emission $(\approx 1.2 \mathrm{keV})$, as expected. The reprocessed component from the non-thermal primary is present in the data, with typical amplitude $R=0.7-1.0$ (Table 3 ). Its presence is however not highly significant, since removing it we obtain fits worse by $\Delta \chi^{2}=5.4-8.4$. The best fit value of ionization parameter is high, $\xi \sim 10^{4}$, as expected from a hot accretion disk, although the formal errors on $\xi$ are large. No further broadening of the reprocessed component is formally required by the data, although the data are consistent with relativistic smearing from a disk extending down to $\sim 20 R_{\mathrm{g}}$. This is larger than the inner radius of the emitting disk, indicating that the innermost disk region is too hot to produce any significant spectral features. A part of the uncertainty in determining $R_{\text {in }}$ comes from the fact that Compton scattering in highly ionized 

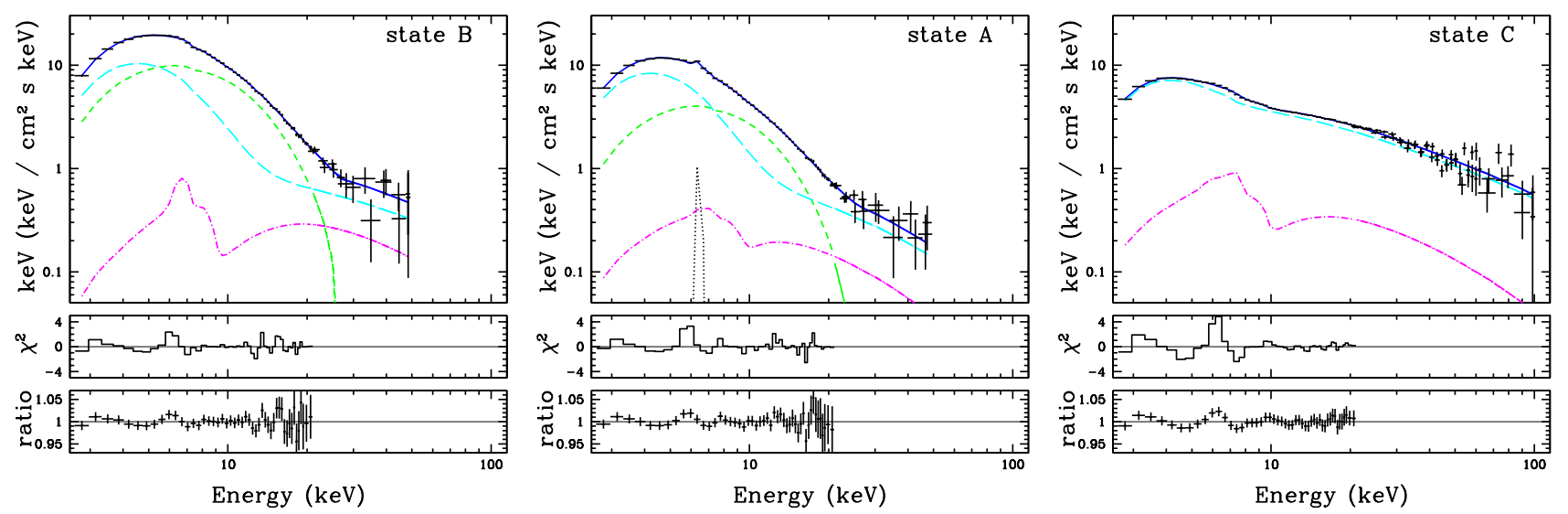

Fig. 1. KerrD model fits to spectra from observation $K$-44-00. Long-dashed curves are the comptonized component compPS with the transmitted seed photons, Short-dashed curves are the Kerr disk spectrum model, and the dash-dot curves are the reprocessed component. Complexity of the soft component in states A and B is apparent and this cannot be accounted for by the Kerr disk model alone. There is only a weak disk thermal component in state $\mathrm{C}$ spectrum, but modeling it with the $\operatorname{Kerr} D$ model suggests that the disk $\dot{M}$ may actually be comparable to or even higher than in states A and B. However, the inner disk radius is larger than the last stable orbit, so most of the accretion power would not appear as thermal $\mathrm{X}$-ray emission.

Table 3. Fit parameters and $\chi^{2}$ values for observation K-44-00 in states $\mathrm{A}, \mathrm{B}$, and C. Model consists of KerrD and non-thermal compPS (A and $\mathrm{B})$, or single compPS (C).

\begin{tabular}{cccc}
\hline \hline Parameter & State B & State A & State C \\
\hline$n_{\mathrm{H}}\left[\times 10^{22} \mathrm{~cm}^{-2}\right]$ & $6.2_{-0.2}^{+0.3}$ & $5.9_{-0.3}^{+0.2}$ & $5.6_{-0.5}^{+0.7}$ \\
\hline$\dot{M}\left[\times 10^{18} \mathrm{gs}^{-1}\right]$ & $7.5_{-3.0}^{+4.5}$ & $1.43_{-0.02}^{+1.50}$ & - \\
$R_{\text {in }}\left[R_{\mathrm{g}}\right]$ & $3.80_{-2.56}^{+2.20}$ & $1.47_{-0.23}^{+2.50}$ & - \\
\hline$k T_{\text {in }}(\mathrm{keV})$ & $1.30_{-0.26}^{+0.13}$ & $1.16_{-0.03}^{+0.03}$ & $0.94_{-0.08}^{+0.06}$ \\
$\Gamma_{\mathrm{e}}$ & $4.8_{-0.9}^{+5.1}$ & $5.9_{-1.7}^{+1.1}$ & $6.7_{-0.5}^{+0.8}$ \\
$\tau$ & $0.05_{-0.05}^{+0.10}$ & $0.06_{-0.04}^{+0.04}$ & $0.44_{-0.22}^{+0.11}$ \\
$R=\frac{\Omega}{2 \pi}$ & $1($ fixed $)$ & $0.70_{-0.58}^{+\infty}$ & $0.30_{-0.15}^{+0.20}$ \\
$\xi$ & $2200_{-2000}^{+17000}$ & $1.10_{-1.02}^{+\infty} \times 10^{4}$ & $6200_{-0000}^{+233000}$ \\
$R_{\text {in,refl }}\left[R_{\mathrm{g}}\right]$ & $165_{-145}^{+\infty}$ & $137_{-131}^{+\infty}$ & $42_{-17}^{+58}$ \\
\hline$\chi_{v}^{2}$ & $0.65\left(\frac{43.3}{67}\right)$ & $0.66\left(\frac{43.8}{66}\right)$ & $1.14\left(\frac{114.5}{101}\right)$ \\
\hline
\end{tabular}

Parameters:

$\dot{M}$ - mass accretion rate in the $\operatorname{Kerr} D$ model.

$R_{\text {in }}$ - inner radius of the disk in the $\operatorname{KerrD}$ model in $R_{\mathrm{g}}=G M / c^{2}$ units.

$k T_{\text {in }}$ - seed photons temperature for Comptonization in $\mathrm{keV}$.

$\Gamma_{\mathrm{e}}-$ index of a power law electron energy distribution.

$\tau$ - Thomson depth of the Comptonizing cloud.

$\Omega / 2 \pi$ - solid angle of the reprocessor.

$\xi=4 \pi F_{\mathrm{X}} / n_{e}-$ ionization parameter of the reprocessor.

$R_{\text {refl }}$ - inner radius of the reprocessing disk.

plasma contributes to smearing of the spectral features, making quantitative determinations of $R_{\text {in }}$ rather uncertain (Ross et al. 1999).

\subsection{State A spectra}

State A is very similar to state B, although somewhat less luminous (B00). The presence of the non-thermal tail was not explicitly demonstrated in this state, but given its similarity to state $\mathrm{B}$, we assume the hard tail is indeed given by a nonthermal Comptonization and include it in all models. Initial fits with the non-thermal compPS model give bad fits $\left(\chi_{v}^{2} \approx 3-5\right)$. Again, a complex shape of soft component helps to obtain good fits, with comparable quality of fits for the various models (Table 2). The overall spectra are dominated by the strong soft component, with the harder tail appearing only above $\approx 20 \mathrm{keV}$ (Fig. 1; Table 3).

The model which includes the Kerr metric disk component gives a comparable fit quality to the other models with a complex soft component. Moreover, the unscattered photons contribute significantly to the soft component, broadening it compared to what would be obtained with pure relativistic disk emission (Fig. 1). Removing the unscatered photons from the model gives clearly unacceptable fits $\left(\chi_{v}^{2} \sim 3\right)$, i.e. the Kerr disk component with pure non-thermal Comptonized component cannot describe the data. In one case $(K-45-03)$ the best overall fit is obtained with the blackbody model while in another case $(K-45-02)$ the Comptonized disk blackbody and the blackbody models provide the same quality of fits to the data. The temperature of the additional blackbody is rather high, $\sim 2 \mathrm{keV}$. It appears that this component is used to adjust the curvature of the Comptonized spectrum rather than representing a real spectral component. Clearly, relativistic effects from a Kerr metric may be considered as contributing to the complexity of the soft component in the case of the $K-44-00$ observation, but are not sufficient to describe it completely.

Other aspects of the best fit models are similar to state B spectra: the reprocessed component is present in the data, it is highly ionized and further smeared (again, with rather large errors). An additional narrow gaussian line at $6.4 \mathrm{keV}$ was also included in the model, since the fit residuals clearly indicated the presence of such a line. Its equivalent width is $\sim 30 \mathrm{eV}$.

Fits of the $\operatorname{KerrD}$ model with the non-thermal tail give $\dot{M}$ systematically lower than those for state $\mathrm{B}$, while $R_{\text {in }}$ is the same within the fit uncertainty (Table 3). 


\subsection{State $C$ spectra}

State $\mathrm{C}$ is generally rather harder than either $\mathrm{A}$ and $\mathrm{B}$, and the disk component is less pronounced (B00). The non-thermal compPS model gives adequate fits to the data (with our assumed systematic errors of $1 \%$ ). The $\chi_{v}^{2}$ values for $K-44-00$, $K$-45-03, $K$-05-00, I-42-00 observations are $\chi_{v}^{2}=1.14,0.83$, 0.87 and 1.04, respectively (Table 2, model 1; Fig. 1).

The disk component can be separated from the total Comptonization component as computed by compPS by setting the un-scattered fraction of seed photons to 0 , and modeling the soft photons by a separate model. We perform such a procedure for the $K-44-00$ and $K-45-03$ data sets, describing the soft component by the KerrD model. Although it does not lead to satisfactory fits, it allows to approximate values of physical disk parameters, $\dot{M}$ and $R_{\text {in. }}$ These are $\dot{M} \approx 2 \times 10^{19} \mathrm{~g} \mathrm{~s}^{-1}$ and $R_{\text {in }} \approx 15 R_{\mathrm{g}}$. The mass accretion rate is rather high, close to the upper values for state $\mathrm{B}$. The large inner radius means that the maximum color temperature is $\approx 0.8 \mathrm{keV}$ and most of the corresponding luminosity is not seen in the X-ray band.

In all data sets the reprocessed component is always significantly present. Its amplitude is smaller than 1, it is highly ionized ( $\xi \sim 10^{4}$, but with large errors). Additional broadening of the spectral features is also required. If this is interpreted as relativistic, the inner radius of the reflecting disk is $\sim 25-100 R_{\mathrm{g}}$ (Table 3).

\section{Discussion}

Our analysis of RXTE data of the micro-quasar GRS 1915+105 reveals all the spectral features typically found in high/soft states of accreting black holes (see Gierliński et al. 1999; Życki et al. 2001; Zdziarski et al. 2001). The hard component is well fit by a non-thermal Comptonization, the soft component is usually complex, and the reprocessed component is present, highly ionized and further broadened and smeared.

A clear complex soft component is seen in the high luminosity states A and B. A number of descriptions of the component are possible: in addition to the usual disk thermal emission, there may be contribution from hotter spots on the disk, heated by strong illumination by compact magnetic flares. This would produce an additional blackbody emission, such as in our model 3. Alternatively, the hot upper layers of the heated accretion disk may additionally comptonize the soft photons from below, producing the second, low temperature comptonized component (see more detailed discussion in, e.g., Życki et al. 2001). However, the relativistic distortions expected from such a highly inclined accretion disk $\left(i \approx 70^{\circ}\right)$ are significant and they cannot be ignored. In particular Doppler blueshift and boosting make the observed temperature higher than the true color disk temperature. However, even assuming a maximally rotating black hole, we need to include a contribution from additional soft photons, in order to explain the spectrum in both state A and B. Therefore, quantitative determinations of the black hole angular momentum are not possible without constructing a more detailed geometrical model explaining the origin of all components. However, relativistic effects alone in Schwarzschild metric $(a=0)$ would not be able to account for the high apparent peak energy of the soft component, if the mass of $14 M_{\odot}$ is adopted. In the interpretation of Zdziarski et al. (2001) the high apparent peak energy is due to Compton upscattering by a relatively cool, optically thick plasma (see Fig. 3b in their paper), but clearly the Comptonization parameters will be changed if relativistic effects are taken into account in this description.

In state $\mathrm{C}$ we generally see strongly comptonized emission with small contribution of soft seed photons. The parameters of the soft component correspond to larger inner disk radius than in states A and B, confirming qualitatively previous studies of Belloni et al. (1997). We note however that quantitative estimates of e.g. the inner disk radius require proper decomposition of spectra into the disk and Comptonized emission. Models used in many previous papers, i.e. diskbb and a power law are clearly inadequate for such a task. In particular a power law is not a proper description of a Comptonization process, if the seed photons are visible in the considered energy band, as is clearly the case in GRS $1915+105$. Our modelling suggests inner radii $\approx 15 R_{\mathrm{g}}$. Since in a Kerr metric the dissipation of gravitational energy is strongly concentrated towards the center, therefore the total accretion power is implied to be much larger than that directly observed as an X-ray emission. Quantitative estimates would require knowledge of the black hole spin, but even for a mild value, $a=0.5$, the fraction of energy dissipated within $15 R_{\mathrm{g}}$ is $\approx 80 \%$. Total accretion power implied would then not be different in state $\mathrm{C}$ compared to states $\mathrm{A}$ and $\mathrm{B}$, but most of that power would be used to e.g. cause an ejection of plasma.

Acknowledgements. This research has made use of data obtained through the High Energy Astrophysics Science Archive Research Center Online Service, provided by the NASA/Goddard Space Flight Center. Partial support was provided by Polish KBN through grant 2P03D01718.

\section{References}

Arnaud, K. A. 1996, Astronomical Data Analysis Software and Systems V, ed. G. Jacoby, \& J. Barnes, ASP Conf. Ser., 101, 17

Belloni, T., Mendez, M., King, A. R., van der Klis, M., \& van Paradijs, J. 1997, ApJ, 488, L109

Belloni, T., Klein-Wolt, M., Mendez M., van der Klis, M., \& van Paradijs, J. 2000, A\&A, 355, 271

Castro-Tirado, A. J., Brandt, S., Lund, N., et al. 1994, ApJS, 92, 469

Coppi, P. S. 1999, in High Energy Processes in Accreting Black Holes, ed. J. Poutanen, \& R. Svensson, ASP Conf. Ser., 161, 375

Done, C., Mulchaey, J. S., Mushotzky, R. F., \& Arnaud, K. A. 1992, ApJ, 395, 275

Ebisawa, K., Kubota, A., Mizuno, T., \& Życki, P. T. 2001, ApSSS, 276,11

Fabian, A. C., Rees, M. J., Stella, L., \& White, N. E. 1989, MNRAS, 238,729

Fender, R. P., Garrington, S. T., McKay, D. J., et al. 1999, MNRAS, 304, 865

Gierliński, M., Maciołek-Niedźwiedzki, A., \& Ebisawa, K. 2001, MNRAS, 325, 1253

Gierliński, M., Zdziarski, A. A., Poutanen, J., et al. 1999, MNRAS, 309, 496 
Greiner, J., Cuby, J. G., \& McCaughrean, M. J. 2001, Nature, 414, 522 Laor, A. 1991, ApJ, 376, 90

Magdziarz, P., \& Zdziarski, A. A. 1995, MNRAS, 273, 837

Martocchia, A., Matt, G., Karas, V., Belloni, T., \& Feroci, M. 2002, A\&A, 387, 215

Merloni, A., Fabian, A. C., \& Ross, R. R. 2000, MNRAS, 313, 193

Mirabel, I. F. \& Rodriguez, L. F. 1994, Nature, 371, 46

Mitsuda, K., Inoue, H., Koyama, K., et al. 1984, PASJ, 36, 741

Nowak, M. A. 1995, PASP, 107, 1207

Poutanen, J., \& Svensson, R. 1996, ApJ, 470, 249

Poutanen, J. 1998, in Theory of Black Hole Accretion Disks, ed. M. A. Abramowicz, G. Björnsson, \& J. Pringle (Cambridge: Cambridge Univ. Press), 100

Ross, R. R., Fabian, A. C., \& Young, A. J. 1999, MNRAS, 306, 461
Shimura, T., \& Takahara, F. 1995, ApJ, 445, 780

Thorne, K. S. 1974, ApJ, 191, 507

Vadawale, S. V., Rao, A. R., \& Chakrabarti, S. K. 2001, A\&A, 372, 793

Vilhu, O., Poutanen, J., Nikula, P., \& Nevalainen, J. 2001, ApJ, 553, 51

Wilson, C. D. \& Done, C. 2001, MNRAS, 325, 167

Zdziarski, A. A., Johnson, W. N., \& Magdziarz, P. 1996, MNRAS, 283, 193

Zdziarski, A. A., Grove, E. J., Poutanen, J., Rao, A. R., \& Vadawale, S. V. 2001, ApJ, 554, L45

Życki, P. T., \& Czerny, B. 1994, MNRAS, 266, 653

Życki, P. T., Done, C., \& Smith, D. A. 1999, MNRAS, 305, 231

Życki, P. T., Done, C., \& Smith, D. A. 2001, MNRAS, 326, 1367 